\title{
Robust Multiple Model Fitting with Preference Analysis and Low-rank Approximation
}

Luca Magri

luca.magri@unimi.it

Andrea Fusiello

andrea.fusiello@uniud.it
Department of Mathematics

Università degli Studi di Milano

Via Saldini, 50 - 20133 Milano, IT

DIEGM

Università degli Studi di Udine

Via delle Scienze, 208 - 33100 Udine, IT
Geometric multi-model fitting aims at extracting parametric models from unstructured data in order to organize and aggregate visual content in suitable higher-level geometric structures. This ubiquitous task can be encountered in many Computer Vision applications, for example in 3D reconstruction, in the processing of 3D point clouds, in face clustering, in body-pose estimation or video motion segmentation, just to name a few.

In practice, it is necessary to overcome the "chicken-\&-egg dilemma" inherent to this problem: in order to estimate models one needs to first segment the data, but in order to segment the data it is necessary to know the models associated with each data point. The presence of multiple structures hinders robust estimation, which has to cope with both gross outliers and pseudo-outliers. Two somehow orthogonal strategies have been proposed in the literature in order to adress this challenging problem: consensus analysis and preference analysis. Consensus based methods, building on the RANSAC paradigm, instantiate a pool of tentative models and extract the strucutures that have maximal consensus. Preference oriented algorithms $[2,3]$ instead tackle this problem by the data point of view. Residuals between point and putative models are used in order to build a conceptual space in which points are portrayed by their preferences with respect to the instantiated strucures, The multi model fitting problem is then solved by clustering points in this preference space.

The method we present reduces the multi-model fitting task to many easier single robust model estimation problems, by combining preference analysis and robust low rank approximation. Three main step can be single out in our appraoch. At first data points are shifted in a conceptual space, where they are framed as a preference matrix $\Phi$ as shown in Fig. 1. Our conceptual representation makes use of an M-estimator in order to

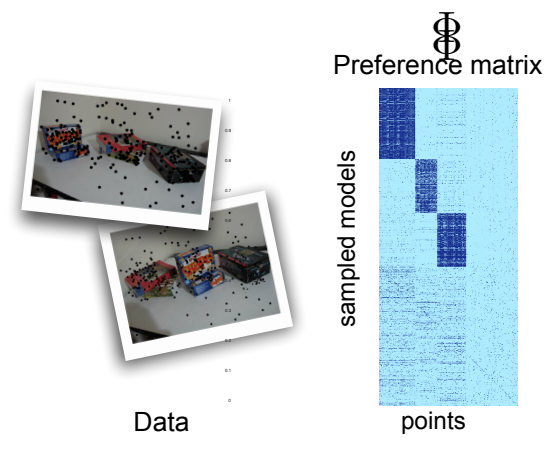

Figure 1: Preference representation

model points preferences, in this way a first protection against outlier is achieved. The preference space is then equipped with a kernel, based on the Tanimoto distance, in this way an affinity matrix $K$, which measures the agreement between the preferences of points, is derived.

The second step is devoted to robustly segment points explointing the information encapsulated in $K$. This stage can be thought as a sort of "robust spectral clustering". It is well known that spectral clustering produces accurate segmentations in two steps: at first data are projected on the space of the first eigenvectors of the Laplacian matrix and then $\mathrm{k}$-means is applied. The shortcoming of spectral clustering however is that it is not robust to outliers. We propose to follow the same scheme enforcing robustness exploiting the low rank nature of the problem. As pictorially illustrated in Fig. 2, we decompose the affinity matrix as

$$
K=U U^{\top}+S
$$

The matrix $S$ models the sparse preferences expressed by outliers, and is obtained by appling Robust PCA, which replaces the eigen-decomposition

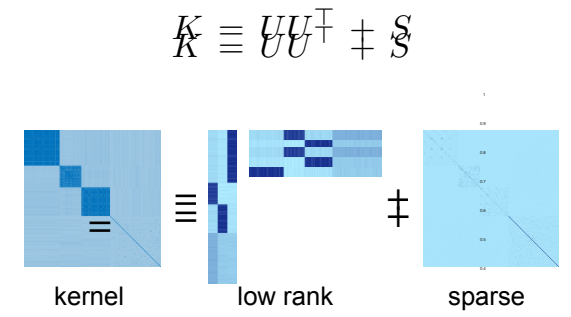

Figure 2: Robust low rank analysis

step of spectral clustering. The low rank part of $K$, representing symilarity between inliers, is hence decomposed as $U U^{\top}$ taking advantage of Symmetric NMF [1], which plays the role of k-means. The obtained matrix $U$ represents a soft segmentation of the data in which outliers are underweighted.

Finally, models are extracted inspecting the product of the preference matrix with a thresholded $U$, mimicking the MSAC strategy. The use of robust statistics for adaptatively estimate the inlier threshold constituites a third guard against the presence of outliers.

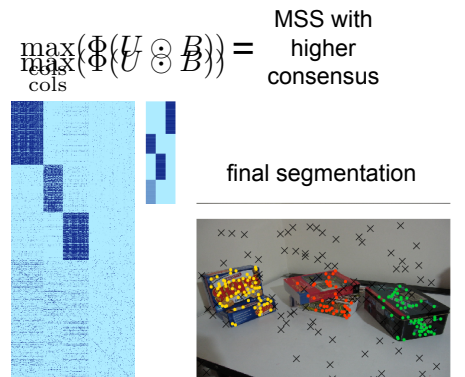

Figure 3: Models and segments extraction

We deal with two applications of geometric multi model fitting on real data: motion segmentation and plane segmentation. In the motion segmentation experiments, given two images of the same scene composed by several objects moving independently, the aim is to fit fundamental matrices to subsets of point matches. In plane segmentation scenario, given two uncalibrated views of a scene, the aim is to recover the multi-planar structures by fitting homographies to point correspondences. The experiments are carried on the AdelaideRMF [4] dataset, composed of 38 image pairs (19 for motion segmentation and 19 for plane segmentation) with matching points corrupted by gross outliers and have provided evidence that our method compares favourably with state of the art competing algorithms.

[1] Da Kuang, Sangwoon Yun, and Haesun Park. Symnmf: nonnegative low-rank approximation of a similarity matrix for graph clustering. Journal of Global Optimization, pages 1-30, 2014.

[2] Luca Magri and Andrea Fusiello. T-linkage: A continuous relaxation of j-linkage for multi-model fitting. In Computer Vision and Pattern Recognition, pages 3954-3961, June 2014.

[3] R. Toldo and A. Fusiello. Robust multiple structures estimation with J-Linkage. In European Conference on Computer Vision, volume 5302, pages 537-547, 2008.

[4] H. S. Wong, T.-J. Chin, J. Yu, and D. Suter. Dynamic and hierarchical multi-structure geometric model fitting. In International Conference on Computer Vision, 2011. 